\title{
Lipidomics Analysis of Peroxisomal Disorders: Discovery of Deficits in Phosphatidyglycerol Levels in Rhizomelic Chondrodysplasia Type 1
}

\section{Paul L Wood ${ }^{1 *}$ and Nancy E Braverman ${ }^{2,3}$}

${ }^{1}$ Metabolomics Unit, Dept. of Physiology and Pharmacology, DeBusk College of Osteopathic Medicine, Lincoln Memorial University, 6965 Cumberland Gap Pkwy., Harrogate, TN 37752, Canada

${ }^{2}$ Department of Human Genetics, McGill University, Quebec, Canada

${ }^{3}$ Department of Pediatrics, Montreal Children's Hospital Montreal, Quebec, Canada

"Corresponing Author: Paul L Wood, Dept. of Physiology and Pharmacology, DeBusk College of Osteopathic Medicine, Lincoln Memorial University, 6965 Cumberland Gap Pkwy, Harrogate, TN 37752, Canada, Tel: 423-869-6666; Fax: 423-869-7174; E-mail: paul.wood@Imunet.edu

Received date: May 08, 2014, Accepted date: June 28, 2014, Published date: June 30, 2014

Copyright: () 2014 Wood PL et al. This is an open-access article distributed under the terms of the Creative Commons Attribution License, which permits unrestricted use, distribution, and reproduction in any medium, provided the original author and source are credited.

\begin{abstract}
Objectives: Decreases in the levels of plasmalogens, have been consistently demonstrated in rhizomelic chondrodysplasia type 1 (RCDP1), a genetic disorder of peroxisomal function. However, an in-depth lipidomics analysis has not been undertaken. We undertook such an analysis.
\end{abstract}

Study Design: We performed a high-resolution mass spectrometric shotgun lipidomics analyses of plasma and lymphoblasts from RCDP1 patients.

Results: We report for the first time, decrements in phosphatidylglycerol levels in plasma and lymphoblasts from RCDP1 patients. Phosphatidylinositol and phosphatidylserine levels also were unaltered in plasma and lymphoblasts. These data suggested that decrements in phosphatidylglycerol were due to increased catabolism, possibly in failed cellular attempts to restore deficient plasmalogen levels. This conclusion was further supported by supplementation of RCDP1 lymphoblasts with ether lipid plasmalogen precursors that bypass dysfunctional peroxisomes. These precursors augmented cellular levels of plasmalogens in control and RCDP1 lymphoblasts but only augmented phosphatidylglycerols in RCDP1 lymphoblasts.

Conclusions: Overall, our results indicate that the peroxisomal disorder, RCDP1, which is characterized by plasmalogen deficits, also possess decrements in phosphatidylglycerol levels, thereby also compromising mitochondrial function and pulmonary surfactant synthesis. Given the role pf phosphatidylglycerols in surfactant, these new data potentially explain the severe respiratory compromise in RCDP children and may add a new parameter of mitochondrial dysfunction in these patients.

Keywords: Phosphatidylglycerol; RCDP; Lung Infections; Lipidomics; High Resolution Mass Spectrometry

\section{Introduction}

Rhizomelic chondrodysplasia punctata type 1 (RCDP1) is a peroxisome biogenesis disorder involving mutations in PEX7, the peroxisome transporter required for the import of enzymes involved in the synthesis of plasmalogens [1-3]. Insertion of a fatty alcohol via an ether linkage at sn-1 of the glycerol backbone of glycerophospholipids only occurs in peroxisomes. Subsequent desaturation of the first carbon-carbon bond takes place in the endoplasmic reticulum, to generate via additional enzymic reactions both mature choline and ethanolamine plasmalogens. Deficiency in these critical glycerophospholipids results in the RCDP1 phenotype, which includes cataracts and shortened humeri and femurs (rhizomelia), puntate epiphyseal calcifications (chondrodysplasia punctate), microcephaly and dysmyelination (hypomyelination) which result in severe growth and neurological impairments.

Plasmalogen levels are routinely monitored in RCDP patients via gas chromatography (GC) and GC-MS assays which involve treating plasma samples with methanol under acidic conditions to yield the dimethylacetal derivatives of the ether linked fatty alcohols at sn-1. This yields a picture of the total plasmalogen pool but does not evaluate individual plasmalogens. We previously have quantitated individual ethanolamine plasmalogens in lymphoblasts from RCDP patients and in a murine Pex7 model of RCDP1 [4]. With this background, we designed studies utilizing high-resolution mass spectrometry to undertake a shotgun lipidomics analysis $[5,6]$ of plasma and lymphoblasts from RCDP1 patients to both more broadly and more precisely evaluate additional lipid alterations in this severe childhood disorder. Since it is the goal of a number of investigators to evaluate plasmalogen precursors as potential therapeutics for peroxisomal disorders $[4,7]$, increasing in our knowledge base of the lipidome in RCDP will be very valuable for future translational research efforts. Furthermore, recognition of a peroxisomal deficit in Alzheimer's disease [8-10], which may underlie cerebral white matter dysmyelination in Alzheimer's disease [10-12], also support increasing our understanding of the full consequences of peroxisomal deficits on the human lipidome. 
Citation: Wood PI, Braverman NE (2014) Lipidomics Analysis of Peroxisomal Disorders: Discovery of Deficits in Phosphatidyglycerol Levels in Rhizomelic Chondrodysplasia Type 1. J Data Mining Genomics Proteomics S1: 001. doi:10.4172/2153-0602.S1-001

Page 2 of 6

\section{Materials and Methods}

\section{Clinical plasma samples}

Plasma samples were obtained from 3 RCDP Caucasian patients aged 1,5 and 6 years, while control plasma was obtained from 8 healthy Caucasian children aged 4 to 8 years. The PEX 7 mutations included, PEX7-p.Leu292* homozygote (c.875T>A); PEX7-Leu292* Tyr40* (c.120C>G); and PEX7-Leu292*/Ala218Val. The clinical studies were approved by the Lincoln Memorial University Institutional Review Board. Plasma samples were processed as described previously, utilizing tert-butyl methylether and methanol for extraction of lipids [4-6,12]. The extraction solution contained $\left[{ }^{2} \mathrm{H}_{8}\right]$ arachidonic acid, $\left[{ }^{2} \mathrm{H}_{3}\right]$ phytanic acid, $\left[{ }^{2} \mathrm{H}_{4}\right]$ hexacosanoic acid, $\left[{ }^{13} \mathrm{C}_{16}\right]$ palmitic acid, $\left[{ }^{2} \mathrm{H}_{7}\right]$ cholesterol sulfate, $\left[{ }^{2} \mathrm{H}_{5}\right]$ MAG 18:1, $\left[{ }^{13} \mathrm{C}_{3}\right]$ DAG 36:2, $\left[{ }^{2} \mathrm{H}_{31}\right]$ PtdEtn 34:1, $\left[{ }^{2} \mathrm{H}_{54}\right]$ PtdEtn 28:0, $\left[{ }^{2} \mathrm{H}_{31}\right]$ PtdCh 34:1, $\left[{ }^{2} \mathrm{H}_{54}\right] \mathrm{PtdCh} 28: 0,\left[{ }^{2} \mathrm{H}_{62}\right] \mathrm{PtdCh} 32: 0,\left[{ }^{2} \mathrm{H}_{31}\right] \mathrm{SM}$ 16:0, PtdSer 36:1, $\left[{ }^{2} \mathrm{H}_{31}\right]$ PA 34:1, $\left[{ }^{2} \mathrm{H}_{62}\right]$ PG 32:0 and $\mathrm{CL}(56: 0)$ as internal standards. Extracts were dried by centrifugal vacuum evaporation and dissolved in isopropanol : methanol : chloroform 4:2:1 containing $7.5 \mathrm{mM}$ ammonium acetate. Shotgun lipidomics were performed utilizing high-resolution (140,000 at $200 \mathrm{amu}$ ) data acquisition, with sub-ppm mass accuracy on an orbitrap mass spectrometer (Thermo Q Exactive) with successive switching between polarity modes [5,6]. Washes between samples with hexane/ethyl acetate (3:2) were used to minimize ghost effects. In negative ion ESI, the anions of ethanolamine plasmalogens, phosphatidylglycerols, phosphatidic acids, phosphatidylinositols, phosphatidylserines, cardiolipins, and fatty acids were quantitated and lipid identities validated by MS/MS $[5,6]$. In positive ion ESI, the cations of choline plasmalogens and the ammonium adducts of diacylglycerols were quantitated and lipid identities validated by MS/MS $[5,6]$

\section{Lymphoblast studies}

Control (GM13072) and RCDP1 (GM09291; Pex7 c.870 871insCAA/875T >A or p.C290 E291insQ/L292X) lymphoblasts were obtained from the Coriell Inst. for Medical Research. Cells were cultured in 12 well plates as described previously [4]. For supplementation studies, batyl alcohol and chimyl alcohol were dissolved in ethanol (final ethanol concentration in culture was $0.2 \%$ ). For precursor labeling studies, lymphoblasts in 12 well plates were incubated with $6 \mathrm{mg} / 100 \mathrm{ml}$ media of either $\left[{ }^{13} \mathrm{C}_{3}\right]$ glycerol or $\left[{ }^{13} \mathrm{C}_{16}\right]$ palmitic acid for 24 hours. Lymphoblasts were harvested by centrifugation at $3,000 \mathrm{xg}$ for $10 \mathrm{~min}$ and washed once with $15 \mathrm{ml}$ of cold PBS. Lipid extraction was the same as for plasma except that the cells were sonicated [4].

\section{Statistical analyses}

Clinical data only involved 3 patients and are therefore presented as vertical scatter plots as \% of control $(\mathrm{N}=8)$. For the lymphoblast studies, data are presented as \% of control [(Tx mean)/(control mean) $\left(1 \pm \operatorname{SQRT}\left((\mathrm{Tx} \mathrm{SD} / \mathrm{Tx} \text { mean })^{2}+(\text { control SD/control mean })^{2}\right)\right.$ for 6 tissue culture wells in all experiments. For the labelling studies, atom percent excess is presented (mean $\pm \mathrm{SD}$ ) after correction for natural isotopic abundance. Data were analyzed by 1-way ANOVA, followed by the Tukey-Kramer test to determine differences between groups.

\section{Results}

\section{RCDP1 plasma lipidomics}

An initial shotgun lipidomics approach demonstrated the expected dramatic decrements in both choline and ethanolamine plasmalogens in the plasma of RCDP1 subjects Figure 1. We also monitored unexpected decreases in the circulating levels of phosphatidylglycerols (PG, Figure 1). While shotgun analyses cannot distinguish between phosphatidylglycerols and bis(monoacylglycero)phosphates (BMP), the decrements in PG 36:1 indicate that the decreases we monitored are mainly constitute decrements the levels of phosphatidylglycerols since BMP 36:1 levels are less than 1\% of the levels of PG 36:1 in human plasma [13].
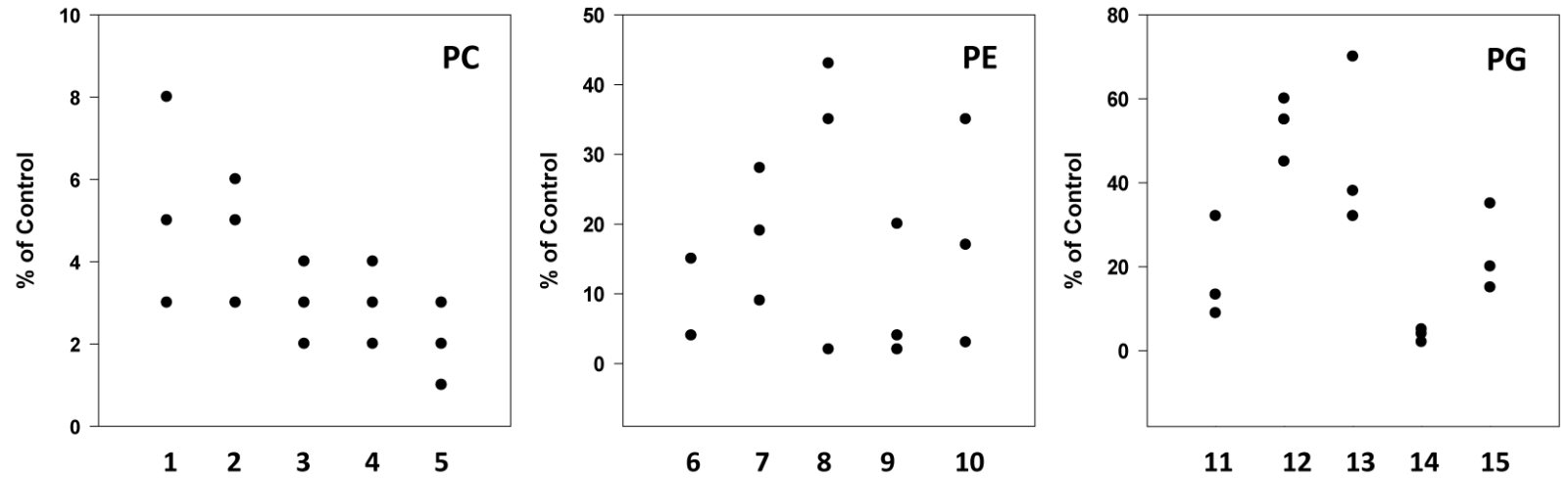

Figure 1: Plasma levels of choline (PC) and ethanolamine (PE) plasmalogens and phosphatidylglycerols (PG). PC 38:6 (1), PC 36:4) (2), PC 34:1 (3), PC 40:6 (4), and PC 38:4 (5) all were decreased (panel PC) in RCDP I subjects (N=3). Data are presented as vertical scatter plots. PE 34:2 (6), PE 36:4 (7), PE 38:6 (8), PE 38:4 (9), and PE 40:6 (10) also were decreased in RCDP plasma (panel PE). Similarly, PG 34:0 (11), PG 34:1 (12), PG 34:2 (13), PG 36:0 (14), and PG 36:1 (15) were lower in RCDP plasma. 
Citation: Wood PI, Braverman NE (2014) Lipidomics Analysis of Peroxisomal Disorders: Discovery of Deficits in Phosphatidyglycerol Levels in Rhizomelic Chondrodysplasia Type 1. J Data Mining Genomics Proteomics S1: 001. doi:10.4172/2153-0602.S1-001

Page 3 of 6

\section{Lipidomics of RCDP1 lymphoblasts}

Next, we took advantage of immortalized cell lines from RCDP1 patients. Using an RCDP1 lymphoblast cell line we validated decrements in plasmalogens and phosphatidylglycerols in cells Figure 2 similar to what we monitored in plasma samples from RCDP1 subjects Figure 1. To further explore other products of the de novo biosynthetic pathway for phosphatidylglycerols Figure 3, we measured phosphatidylinositols and found that the levels of these glycerophospholipids were not decreased Figure 2. Phosphatidic acids, which are at a critical branchpoint interconnecting multiple glycerophospholipid pathways Figure 3 were elevated Figure 2. The very-long-chain fatty acid (VLCFA), octacosanoic acid (26:0) also was elevated Figure 2 in RCDP lymphoblasts, another biomarker of peroxisomal dysfunction since this is the compartment where metabolism of VLCFA is initiated.

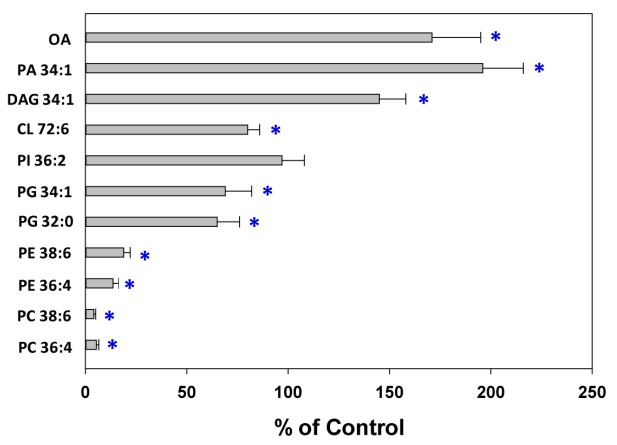

Figure 2: Glycerophospholipid and very-long-chain fatty acid levels in RCDP I lymphoblasts. The VLCFA, octacosanoic acid (OA), phosphatidic acid (PA 34:1), and diacylglycerol (DAG 34:1) were significantly elevated in RCDP lymphoblasts relative to control cells. Data are presented as \% of control \pm SD for 6 tissue culture wells. Phosphatidylinositols (PI 34:1) were not altered while significant decrements in the levels of phosphatidylglycerols (PG 32:0 and PG 34:1), ethanolamine plasmalogens (PE 36:4 and PE 38:6), choline plasmalogens (PC 36:4 and PC 38:6), and cardiolipins (CL 72:6) were measured. ${ }^{*}, \mathrm{p}<0.05$.

\section{Glycerophospholipid biosynthesis in lymphoblasts}

To monitor the dynamics of phosphatidylglycerol synthesis, we utilized stable isotope precursors [14]. In our first experiment, we utilized $\left[{ }^{13} \mathrm{C}_{16}\right]$ palmitic acid to label glycerophospholipid pools in lymphoblasts. Incorporation into plasmalogens Figure 4, panel A was drastically decreased in RCDP lymphoblasts compared to control lymphoblasts, as predicted by previous labeled precursor studies [4]. In contrast the incorporation of [13C16]palmitic acid into phosphatidylglycerols Figure 4, panel Bwas not different from controls. [13C16]Palmitic acid incorporation into DAG 34:1 Figure 2, panel B was increased $(\mathrm{p}<0.01)$ while incorporation into DAG $(32: 0)$ was not different from control cells (data not shown).

Next, we evaluated $\left[{ }^{13} \mathrm{C}_{3}\right]$ glycerol incorporation into lipid pools Figure 4, panel C. With this precursor, incorporation rates as percent of the pool were unaltered for plasmalogens (PE and PC) as well as for phosphatidylserines. Increased incorporation into phosphatidylglycerols was monitored in RCDP lymphoblasts.

\section{Phosphatidyglycerol metabolism}

The data that we obtained for the steady-state levels and biosynthesis of phosphatidylglycerols led us to conclude that these lipids might be catabolized in a failed effort to supply precursors for plasmalogen synthesis. With this hypothesis in mind we first measured cardiolipins (CL 72:5, CL 72:6, and CL 77:8) in lymphoblasts and diacylglycerols (DAG 34:1, DAG 36:4, and DAG 34:1) in both lymphoblasts and patient plasma samples. In lymphoblasts, cardiolipins were decreased by 20 to $30 \%$ while diacylglycerols were increased by 30 to $50 \%$ Figure 2 . These data were consistent with the initial hypothesis; however, diacylglycerols were not increased in patient's plasma (data not shown).

\section{Ether lipid supplementation}

To further evaluate if increased catabolism of phosphatidylglycerols was potentially the result of failed attempts to restore plasmalogens via the metabolism of phosphatidylglycerols to augment diacylglycerol levels Figure 3, we investigated augmentation of plasmalogens with the ether lipid precursors, batyl alcohol and chimyl alcohol, which bypass peroxisomes in the synthesis of plasmalogens $[7,15]$. Chimyl alcohol was the most effective in augmenting a broad range of choline plasmalogens Figure 5, upper panel and ethanolamine plasmalogens (data not shown) in both control and RCDP1 lymphoblasts. While these increases in cellular plasmalogen levels did not alter phosphatidylglycerol levels in control lymphoblasts, this treatment did increase the levels of phosphatidylglycerols (32:0 and 32:1) in RCDP1 lymphoblasts Figure 5, lower panel. These are predominant phosphatidylglycerols involved in surfactant synthesis in humans [16-18].

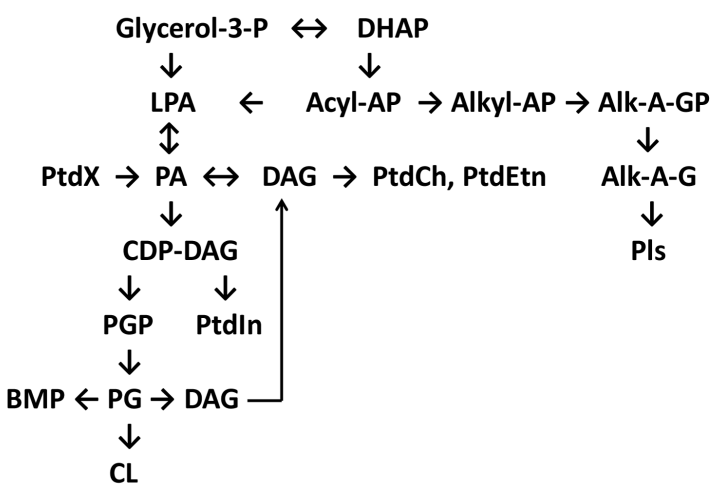

Figure 3: Schematic of the de novo cytidine diphosphatediacylgycerol (CDP-DAG) biosynthetic pathway for phosphatidylglycerols (PG). BMP, bis(monoacylglycero)phosphate; CL, cardiolipin; DHAP, dihydroxyacetone phosphate; $\mathrm{P}$, phosphate; PA, phosphatidic acid (lysophosphatidylglycerol); PtdEtn, phosphatidylethanolamines; PtdCh, phosphatidylcholines; PtdIn, phosphatidylinositol; PtdSer, phosphatidylserine. 
Citation: Wood PI, Braverman NE (2014) Lipidomics Analysis of Peroxisomal Disorders: Discovery of Deficits in Phosphatidyglycerol Levels in Rhizomelic Chondrodysplasia Type 1. J Data Mining Genomics Proteomics S1: 001. doi:10.4172/2153-0602.S1-001

Page 4 of 6

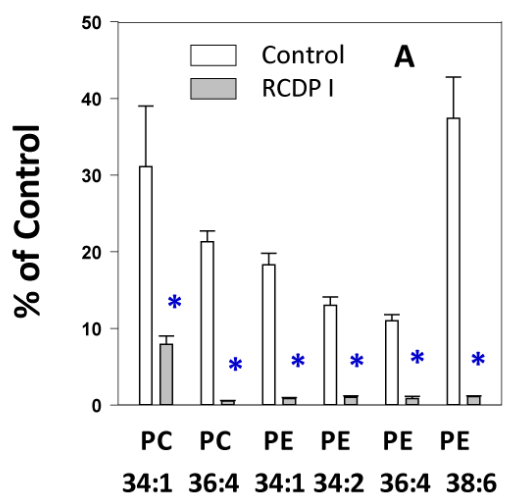

34:1 36:4 34:1 34:2 36:4 38:6

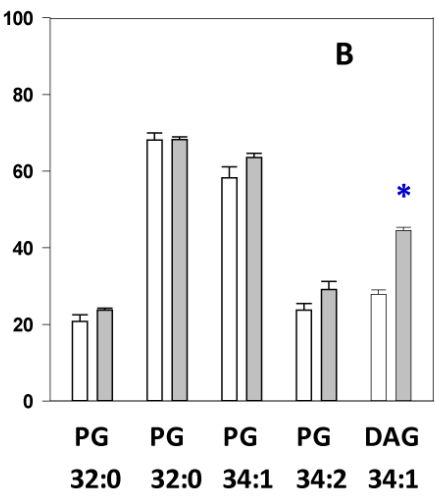

(1) (2)

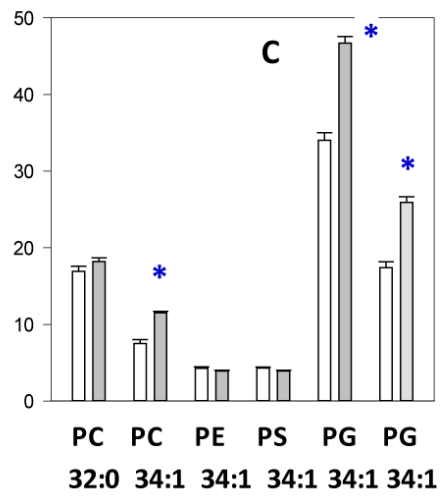

(1) (2)

Figure 4: Glycerophospholipid synthesis in control and RCDP I lymphoblasts. Cellular choline plasmalogens (PC), ethanolamine plasmalogens (PE), phosphatidylglycerols (PG), diacylglycerols (DAG), and phospahtidylserines (PS) labeling with $200 \mu \mathrm{M}$ of the precursors $\left[{ }^{13} \mathrm{C}_{16}\right]$ palmitic acid (panels $\mathrm{A}$ and $\mathrm{B}$ ) or $\left[{ }^{13} \mathrm{C}_{3}\right]$ glycerol (panel $\mathrm{C}$ ), expressed as atom percent excess (APE) incorporation of stable isotope label over 24 hours in serum free media. The (1) and (2) symbols are indicative of the number of incorporated $\left[{ }^{13} \mathrm{C}_{16}\right]$ palmitic acid residues (panel $\mathrm{B}$ ) or $\left[{ }^{13} \mathrm{C}_{3}\right]$ glycerol residues (panel C). Data are expressed as mean $\pm \mathrm{SD}$ for 6 tissue culture wells. Labeling of plasmalogens (panel A) with $\left[{ }^{13} \mathrm{C}_{16}\right]$ palmitic acid was significantly decreased in RCDP cells, while that of DAG 34:1 was augmented, and labeling of phosphatidylglycerols was not different from control cells (panel B). Labeling of plasmalogens and phosphatidylserine with $\left[{ }^{13} \mathrm{C}_{3}\right]$ glycerol was not different between controls and RCDP cells, while phosphatidylglycerol synthesis [PG 34:1 (1) and PG 34:1 (2)] was significantly increased in RCDP cells (panel C). ${ }^{\star}, \mathrm{p}<0.05$ vs. control.

\section{Discussion}

A key factor that negatively impacts the quality of lifestyle and ultimately limits longevity in RCDP1 patients is respiratory compromise with frequent lung infections and reactive airway disease $[19,20]$. In this regard, our observations of decreased phosphatidyglycerol levels in the plasma and lymphoblasts of RDDP1 patients is of significant notation. Phosphatidylglycerols are precursors to mitochondrial cardiolipins [21] and are essential components of lung surfactant $[22,23]$. ToF-SIMS studies have demonstrated that phosphatidylglycerols concentrate along the edges of the tubular proteolipids that form the tubular myelin of pulmonary surfactant and are dispersed throughout the interstitial space between the tubular networks [24]. Of further significance are the observations that phosphatidylglycerols regulate innate immunity against lung viral infections and the associated inflammatory processes [23-31]. Recent studies have also demonstrated in murine models that phosphatidylglycerol administration is effective both for postinfection treatment and for prophylaxis against respiratory syncytial viral infections [32], a predominant respiratory pathogen in young children. This combination of phosphatidylglycerol alterations in surfactant, the innate immune response, and mitochondrial function may contribute significantly to the incidence of respiratory infections in RCDP1 children.

Phosphatidylglcerols are the second most abundant phospholipids that are constituent in surfactant. The dominant phospholipid is dipalmitoylphosphatidylcholine, a glycerophospholipid that was not decreased in plasma or lymphoblasts from RCDP1 patients, in our study. In contrast, we observed decreases in the levels of the major surfactant phosphatidyglycerol PG 34:1 [17] in both plasma and lympoblasts from RCDP1 subjects. Since we observed no decrements in phosphatidylinositol levels nor in the incorporation of labeled glycerol or palmitic acid into phosphatiylglycerol pools,we conclude that the de novo CDP-DAG biosynthetic pathway [23] and acylation / transacylation remodeling of phosphatidylglyverols [33] are operational in RCDP1. The labeling with $\left[{ }^{13} \mathrm{C}_{3}\right]$ glycerol also indicate that aquaporin channels are functional in RCDP1 and do not limit cellular access of glycerol [34].

The decreases in RCDP1 lymphoblasts of cardiolipins along with increases in diacylglycerols and in the synthesis of DAG 34:1, a major precursor of phosphatidylglycerols in surfactant, further suggest that phosphatidylglycerol catabolism is accelerated in RCDP1. This conclusion is also consistent with previous observations that lung phosphatidylglycerols are more susceptible to degradation and recycling than phosphatidylcholines [33]. While we did not monitor decreases in circulating levels of diacylglycerols in RCDP1 subjects, these measurements may be complicated by the complex role of diacylglycerols in the synthesis and metabolism of a vast array of glycerolipids and glycerophospholipids in multiple compartments [23].

In toto, our data led us to hypothesize that phosphatidylglycerol catabolism is augmented in RCDP1 in a failed metabolic attempt to augment cellular plasmalogens via increasing the diacylglycerol precursor pool. To evaluate this hypothesis we investigated augmentation of cellular plasmalogens in lymphoblasts incubated with ether lipid precursors that bypass peroxisomes to augment cellular plasmalogen levels. These data suggest that ether lipid supplementation to augment plasmalogens $[7,15]$ in RCDP1 patients also may augment phosphatidylglycerols. If this proves to be the case, then improved quality of lifestyle and longevity, relative to lung infections, may be a significant clinical benefit for RCDP children. 
Citation: Wood PI, Braverman NE (2014) Lipidomics Analysis of Peroxisomal Disorders: Discovery of Deficits in Phosphatidyglycerol Levels in Rhizomelic Chondrodysplasia Type 1. J Data Mining Genomics Proteomics S1: 001. doi:10.4172/2153-0602.S1-001

Page 5 of 6

\section{Acknowlegements}

We are grateful to the parents who supplied the blood samples for their children with RCDP1 and to parents who have contributed to establishing patient lymphoblast cell lines via the Coriell Inst. We also thank Rhizo Kids International for supporting this research.
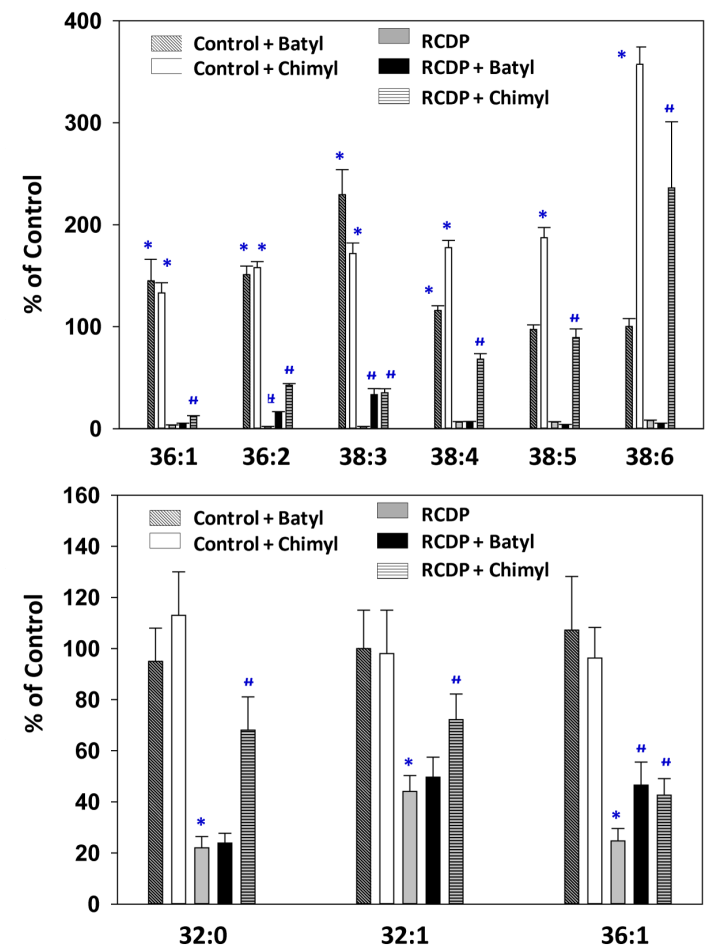

Figure 5: Glycerophospholipid levels in control and RCDP I lymphoblasts with ether lipid supplementation. Cellular choline plasmalogens (upper panel) and phosphatidylglycerols (lower panel) in control and RCDP1 lymphoblasts after a $24 \mathrm{hr}$ incubation with vehicle, $50 \mu \mathrm{M}$ batyl alcohol, or $50 \mu \mathrm{M}$ chimyl alcohol. Chimyl alcohol significantly augmented choline plasmalogens in both control and RCDP1 lymphoblasts, while batyl alchol was less effective (upper panel). While ether lipid precursors had no effect on phosphatidylglycerol levels in control cells, augmentation of plasmalogens with chimyl alcohol in RCDP1 cells resulted in significant increases in cellular PG 32:0, PG 32:1, and PG 36:1 levels (lower panel). Batyl alcohol significant increased cellular levels of PG 36:1 (lower panel). ${ }^{*}, \mathrm{p}<0.05$ vs. control; \#, $\mathrm{p}<0.05$ vs. RCDP1.

\section{Funding}

These studies were funded by Rhizo Kids International (to P.L.Wood). The funding agency had no role in the design of the study, in data collection, or data analysis, decision to publish, or preparation of the manuscript.

\section{Competing Interests}

The authors have no declared competing interests.

\section{References}

1. Aubourg P, Wanders R (2013) Peroxisomal disorders. Handb Clin Neurol 113: 1593-1609.

2. Fidaleo M (2010) Peroxisomes and peroxisomal disorders: the main facts. Exp Toxicol Pathol 62: 615-625.

3. Braverman NE, D'Agostino MD, Maclean GE (2013) Peroxisome biogenesis disorders: Biological, clinical and pathophysiological perspectives. Dev Disabil Res Rev17: 187-196.

4. Wood PL, Khan MA, Smith T, Ehrmantraut G, Jin W, et al. (2011) In vitro and in vivo plasmalogen replacement evaluations in rhizomelic chrondrodysplasia punctata and Pelizaeus-Merzbacher disease using PPI-1011, an ether lipid plasmalogen precursor. Lipids Health Dis 10: 182.

5. Schuhmann K, Almeida R, Baumert M, Herzog R, Bornstein SR, et al. (2012) Shotgun lipidomics on a LTQ Orbitrap mass spectrometer by successive switching between acquisition polarity modes. J Mass Spectrom 47: 96-104.

6. Wood PL, Shirley NR (2013) Lipidomics analysis of postmortem interval: Preliminary evaluation of human skeletal muscle. Metabolomics 3: 127.

7. Wood PL (2013) Alkylglycerol Lipid precursors: A review of therapeutic strategies for plasmalogen replacement. Current Organic Chemistry 17: 786-792.

8. Astarita G, Jung KM, Berchtold NC, Nguyen VQ, Gillen DL, et al. (2010) Deficient liver biosynthesis of docosahexaenoic acid correlates with cognitive impairment in Alzheimer's disease. PLoS One 5.

9. Kou J, Kovacs GG, Höftberger R, Kulik W, Brodde A, et al. (2011) Peroxisomal alterations in Alzheimer's disease. Acta Neuropathol 122: 271-283.

10. Wood PL (2012) Lipidomics of Alzheimer's disease: current status. Alzheimers Res Ther 4: 5.

11. Baes M, Aubourg P (2009) Peroxisomes, myelination, and axonal integrity in the CNS. Neuroscientist 15: 367-379.

12. Wood PL, Mankidy R, Ritchie S, Heath D, Wood JA, et al. (2010) Circulating plasmalogen levels and Alzheimer Disease Assessment ScaleCognitive scores in Alzheimer patients. J Psychiatry Neurosci 35: 59-62.

13. Meikle PJ, Duplock S, Blacklock D, Whitfield PD, Macintosh G, et al. (2008) Effect of lysosomal storage on bis(monoacylglycero)phosphate. Biochem J 411: 71-78.

14. Postle AD, Hunt AN (2009) Dynamic lipidomics with stable isotope labeling. J Chromatogr B Analyt Technol Biomed Life Sci 877: 2716-2721.

15. Braverman N, Zhang R, Chen L, Nimmo G, Scheper S,et al. (2010) A Pex7 hypomorphic mouse model for plasmalogen deficiency affecting the lens and skeleton. Mol Genet Metab 99: 408-416.

16. Goss V, Hunt AN, Postle AD (2013) Regulation of lung surfactant phospholipid synthesis and metabolism. Biochim Biophys Acta 1831: 448-458.

17. Okano G, Sato T, Akino T (1981) Biosynthetic routes of molecular species of lung phosphatidylglycerol. Tohoku J Exp Med 135: 265.

18. Postle AD, Heeley EL, Wilton DC (2001) A comparison of the molecular species compositions of mammalian lung surfactant phospholipids. Comp Biochem Physiol A Mol Integr Physiol 129: 65-73.

19. White AL, Modaff P, Holland-Morris F, Pauli RM (2003) Natural history of rhizomelic chondrodysplasia punctate. Am J Med Genet A 118A: 332-342.

20. Braverman NE, Moser AB, Steinberg SJ (2012) Rhizomelic Chondrodysplasia Punctata Type 1. In: GeneReviews ${ }^{\mathrm{m}}$ [Internet] Pagon RA, Adam MP, Bird TD, Dolan CR, Fong CT, et al. (Eds). University of Washington, Seattle.

21. Claypool SM, Koehler CM (2012) the complexity of cardiolipin in health and disease. Trends Biochem Sci 37: 32-41.

22. Akella A, Deshpande SB (2013) pulmonary surfactants and their role in pathophysiology of lung disorders. Indian J Exp Biol 51: 5-22. 
Citation: Wood PI, Braverman NE (2014) Lipidomics Analysis of Peroxisomal Disorders: Discovery of Deficits in Phosphatidyglycerol Levels in Rhizomelic Chondrodysplasia Type 1. J Data Mining Genomics Proteomics S1: 001. doi:10.4172/2153-0602.S1-001

Page 6 of 6

23. Agassandian M, Mallampalli RK (2013) Surfactant phospholipid metabolism. Biochim Biophys Acta 1831: 612-625.

24. Keating E, Waring AJ, Walther FJ, Possmayer F, Veldhuizen RA, et al. (2011) A ToF-SIMS study of the lateral organization of lipids and proteins in pulmonary surfactant systems. Biochim Biophys Acta1808 614-621.

25. Chiba H, Piboonpocanun S, Mitsuzawa H, Kuronuma K, Murphy RC, et al. (2006) Pulmonary surfactant proteins and lipids as modulators of inflammation and innate immunity. Respirology.

26. Kuronuma K, Mitsuzawa H, Takeda K, Nishitani C, Chan ED, et al (2009) Anionic pulmonary surfactant phospholipids inhibit inflammatory responses from alveolar macrophages and U937 cells by binding the lipopolysaccharide-interacting proteins CD14 and MD-2. J Biol Chem 284: 25488-25500.

27. Numata M, Chu HW, Dakhama A, Voelker DR (2010) Pulmonary surfactant phosphatidylglycerol inhibits respiratory syncytial virusinduced inflammation and infection. Proc Natl Acad Sci U S A 107: 320-325.

28. Kandasamy P, Zarini S, Chan ED, Leslie CC, Murphy RC, et al. (2011) Pulmonary surfactant phosphatidylglycerol inhibits Mycoplasma pneumoniae-stimulated eicosanoid production from human and mouse macrophages. J Biol Chem 286: 7841-7853.

29. Numata M, Kandasamy P, Nagashima Y, Posey J, Hartshorn K, et al. (2012) Phosphatidylglycerol suppresses influenza A virus infection. Am J Respir Cell Mol Biol 46: 479-487.

30. Numata M, Kandasamy P, Voelker DR (2012) Anionic pulmonary surfactant lipid regulation of innate immunity. Expert Rev Respir Med 6: 243-246.

31. Perino J, Crouzier D, Spehner D, Debouzy JC, Garin D, et al. (2011) Lung surfactant DPPG phospholipid inhibits vaccinia virus infection. Antiviral Res 89: 89-97.

32. Numata M, Nagashima Y, Moore ML, Berry KZ, Chan M, et al. (2013) Phosphatidylglycerol provides short-term prophylaxis against respiratory syncytial virus infection. J Lipid Res 54: 2133-2143.

33. Agre P (2004) Nobel Lecture Aquaporin water channels. Biosci Rep 24: 4278-4290.

34. Quintero OA, Wright JR (2010) Metabolism of phosphatidylglycerol by alveolar macrophages in vitro. Am J Physiol Lung Cell Mol Physiol 279: 399-407.
This article was originally published in a special issue, entitled: "Systems Biology : Biomarker Discovery", Edited by Dr. Firas Kobeissy, University of Florida, USA 